\title{
Studies on Aconitine-induced Arrhythmias
}

\section{The Ectopic Pacemaker Formation in the Left Superior Vena Cava Proximal to the Heart and the Genesis of Cardiac Arrhythmias}

\author{
Takasi Yanaga, M.D., Morio Ito, M.D., Kazuyuki Saeki, M.D., Makoto \\ Arita, M.D., Mitsuo Tanoue, M.D., and Hiroto Mashiba, M.D.
}

Aconitine induced the automatic activities in the isolated strip of the left superior vena cava in rabbit. In the left superior vena cava near the coronary sinus, action potentials with diastolic slow depolarization were recorded after the application of aconitine. When aconitine was applied to the left superior vena cava near the coronary sinus in situ, cardiac arrhythmias were recorded. From the above-mentioned facts, it was suggested that aconitine induced the ectopic pacemaker in the left superior vena cava proximal to the heart and that the impulse from the ectopic focus spread not only to the periphery but also to the atrium. In the patient with persistent left superior vena cava the ectopic pacemaker formation in the vena caval muscle may have the significance for the genesis of cardiac arrhythmias, especially supraventricular extrasystoles.

\begin{abstract}
AONITINE is a characteristic substance which induces cardiac arA rhythmias. When locally applied to the cardiac muscle, it induces ectopic pacemakers at the area of application. However, the ectopic pacemaker formation in the left superior vena cava proximal to the heart has never been reported. Recently, it was found that the impulse which originated in the sinus node spread not only to atrial muscle but also to vascular muscle in vena cava (sino-caval conduction), ${ }^{11-3)}$ that the configuration of transmembrane potential of left superior vena caval muscle had close similarity to that of cardiac muscle, ${ }^{4)}$ and that the contractile tension could be recorded. ${ }^{5}$. In connection with these facts, the ectopic pacemaker formation by aconitine in the left superior vena cava proximal to the heart was studicd in rabbit.
\end{abstract}

\section{MetHODS}

The rabbits weighing 1.5 to $2 \mathrm{Kg}$. were used. After intraperitoneal injection of pentobarbital sodium, the animals were thoracotomized. Experiments were carried out in vitro and in situ. In the in vitro experiment, after three venae cavae were cut at $2-3 \mathrm{~cm}$. from their cardiac orifices, the heart was excised. Then a large

From the First Department of Internal Medicine (Director: Prof. K. Yamaoka), Faculty of Medicine, Kyushu University, Fukuoka, Japan.

Received for publication March 17, 1966. 
part of both ventricles were cut away. This atrio-caval preparation was so fixed that the dorsal side faced upward and that each vena cava was fixed at the in situ length, and it was irrigated with oxygenated Tyrode solution at a constant flow and constant temperature $\left(37^{\circ} \mathrm{C}\right)$. The isolated strip of left superior vena cava was also used. The transmembrane potentials were recorded from the external surface of the vena cava. For inducing the fibrillation, a $0.1 \%$ solution of aconitine was instilled in the chamber after stopping the flow of perfusate to make the final concentration of $10^{-5} \mathrm{Gm} . / \mathrm{ml}$. In the in situ experiment, the pericardium was excised after thoracotomy. The heart was positioned the dorsal side faced upward to fully expose the left superior vena cava. For inducing the cardiac arrhythmias, a piece of cotten soaked by a few drops of $0.1 \%$ solution of aconitine was applied to the left superior vena cava near the coronary sinus. The lead II electrocardiogram was recorded.

\section{Results}

1. Induction of the automatic activities of the isolated vena caval strip by aconitine.
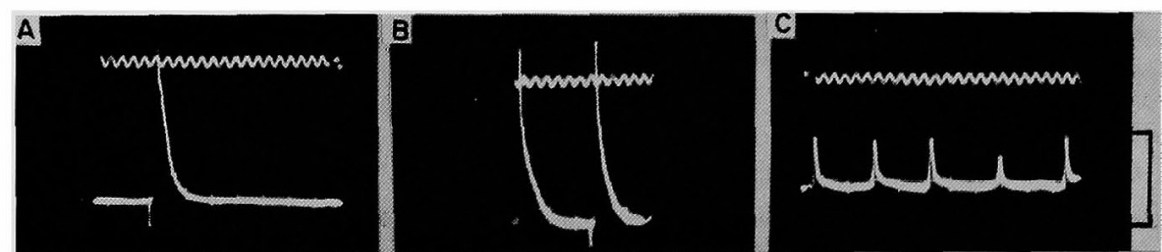

Fig. 1. Induction of the automatic activities in the isolated vena caval strip by aconitine. A : Before the application of aconitine. B : A few min. after the application of aconitine. Note the onset of spontaneous firing. $\mathrm{C}$ : Note the appearance of typical fibrillation. Calibrations represent $20 \mathrm{cps}$ and $50 \mathrm{mV}$.

Fig. 1 shows the induction of fibrillation in the isolated strip of vena cava by aconitine. The strip was electrically driven at the rate of 90 per min. (A). A few min. after the application of aconitine, spontaneous firing appeared intermittently and it became more and more frequent (B). Then, the electrical stimulation was stopped. The isolated strip continued the electrical activity of high frequency and finally the typical fibrillation developed. During this period, action potentials of various magnitudes appeared quite irregularly $(\mathrm{G})$.

2. Transmembrane potentials recorded from the aconitine-induced ectopic pacemaker cells.

If aconitine induces ectopic pacemakers in the left superior vena cava, the action potentials with diastolic slow depolarization could be recorded. Such pacemaker potentials were frequently recorded during recovery from aconitine poisoning. When aconitine in high concentrations $10^{-5} \mathrm{Gm} . / \mathrm{ml}$. was administered, fibrillation continued only a few min. and all activities ceased. 
However, when perfusion with normal Tyrode solution was reinstituted, the gradual appearance of activity was observed.

Fig. 2 shows the record obtained in this period. This was recorded from the left superior vena cava near the coronary sinus in the atrio-caval preparation. As seen in this record, the electrical activity was usually intermittent, waxing and waning alternately. Such alternative activity of the muscle was also observed microscopically. After this period, typical fibrillation developed.

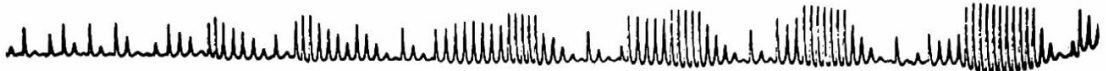

Fig. 2. Waxing and waning potentials recorded from the region of the left superior vena cava near the coronary sinus. Calibrations represent $10 \mathrm{sec}$. and $50 \mathrm{mV}$. See text for further explanation.
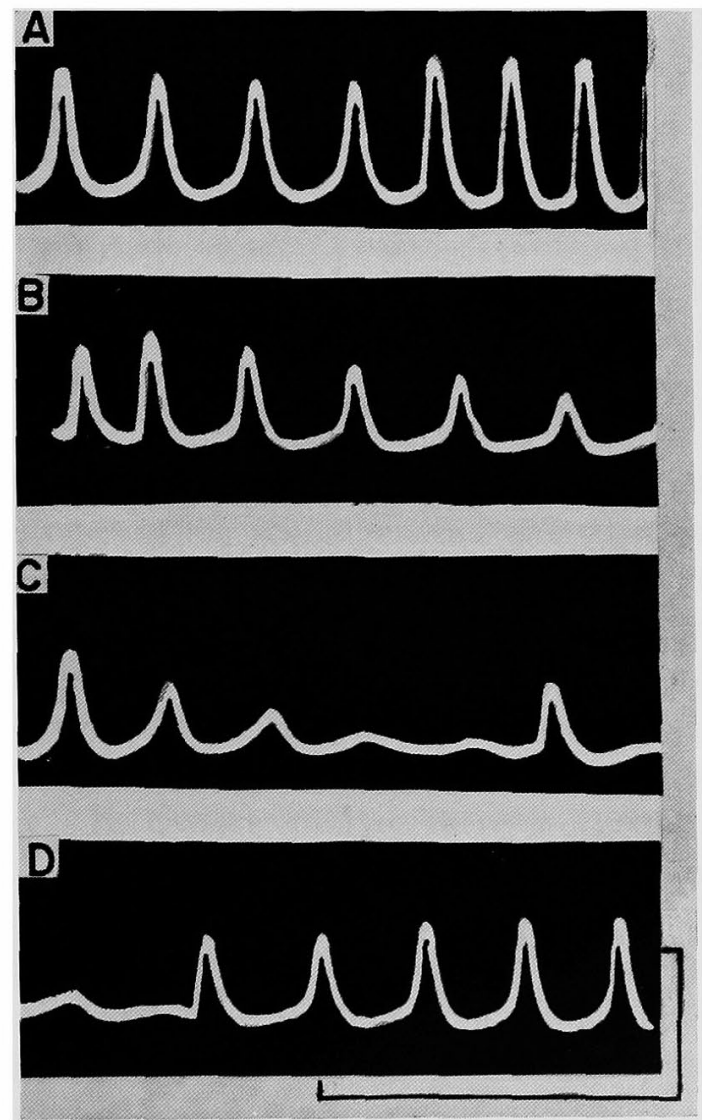

Fig, 3. Series of photographic records of the waxing and waning potentials shown in Fig. 3. Note the diastolic slow depolarizations. Calibrations represent 1 sec. and $50 \mathrm{mV}$. Explanation is in text. 
Fig. 3 shows the series of photographic records obtained during the waxing and wanig phenomena. Diastolic slow depolarizations were clearly observable.

On the other hand, when the muscle contraction was not visible, oscillations in membrane potential were recorded at times. Fig. 4 shows such oscillations in membrane potential recorded from the region of the left superior

A

B

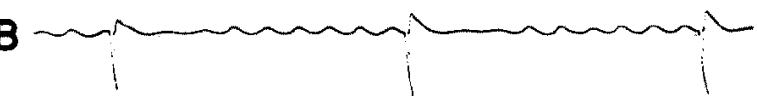

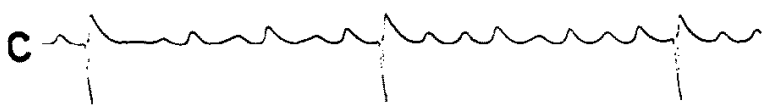

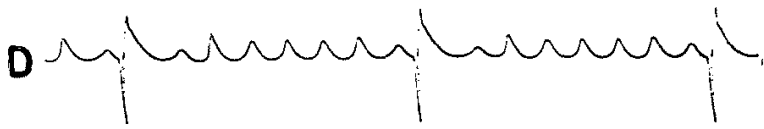

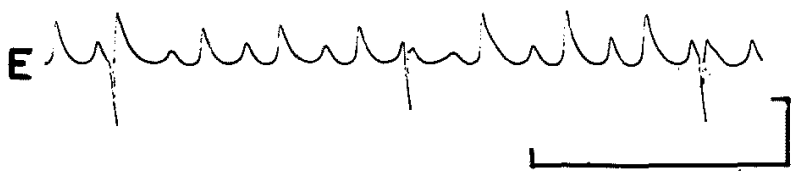

Fig. 4. Oscillatory potentials recorded from the left superior vena caval muscle $10 \mathrm{~mm}$. from the cardiac orifice. Calibrations represent 1 sec. and $50 \mathrm{mV}$.
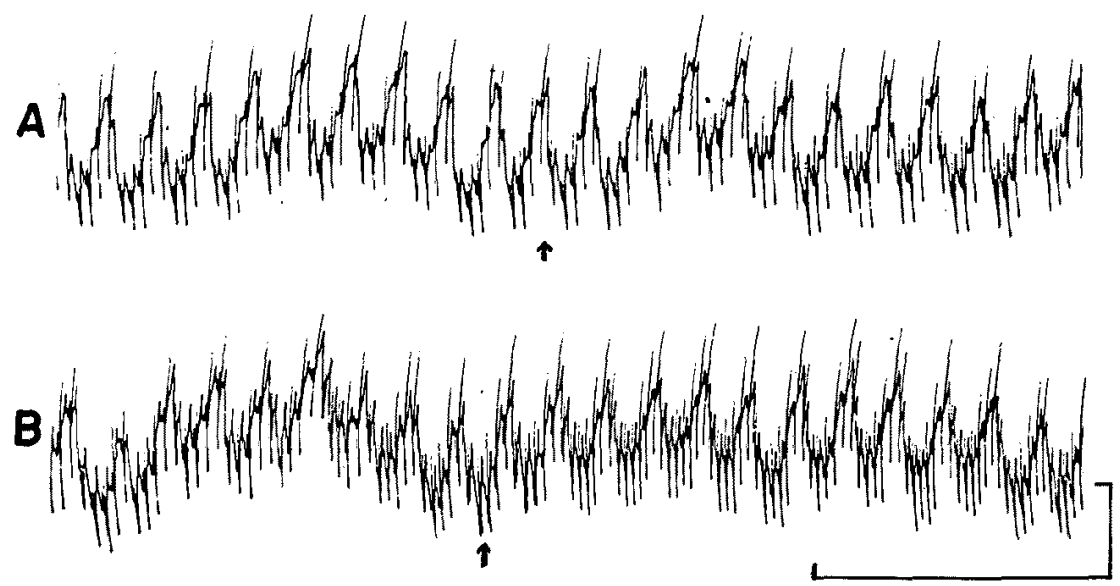

Fig. 5. Tachycardia induced by the local application of aconitine to the left superior vena caval muscle proximal to the heart. Arrow in the upper trace shows aconitine application and that in lower trace, onset of tachycardia. Calibrations represent $500 \mu \mathrm{V}$. and $10 \mathrm{sec}$. See text for further explanation. 
vena cava $10 \mathrm{~mm}$. from the cardiac orifice (Fig. 4). These oscillatory potentials consisted of spontaneous regular slow waves (A). If further electrical stimulation was not given, these potentials became smaller and smaller and finally ceased. When electrical stimulation was applied repeatedly, these potentials, as shown in $\mathrm{B}$ to $\mathrm{D}$, increased in amplitude and reached the firing level with the appearance of spike. Lastly, as shown in E, typical fibrillation developed.

3. Induction of tachycardias by local application of aconitine to the left superior vena caval muscle in situ.

From the above-mentioned results, it would be presumed that ectopic pacemaker can develop in the vena caval muscle. Then, the experiment was carried out in order to determine whether or not the impulse from the ectopic focus spread to the atrium. A few min. after the local application of aconitine to the left superior vena cava near the coronary sinus, tachycardia appeared (Fig. 5).

\section{Discussion}

The principal findings reported here are as follows: (1) The ectopic pacemaker can develop in the left superior vena cava proximal to the heart, (2) The impulse from the ectopic focus spreads not only to the periphery but also to the atrium. That ectopic pacemaker can develop in the vena cava was suggested from the facts that the automatic activities of the vena caval muscle was induced in the isolated strip and the action potentials with diastolic slow depolarization were recorded in the vena caval muscle after the application of aconitine.

There were 2 types of the aconitine-induced ectopic pacemaker cells in the vena cava. One was an oscillatory potential and the other was a waxing and waning potential. The former is similar to the oscillatory potential observed by Matsuda in ventricular muscle, $\left.{ }^{6}\right)$ and the latter, the waxing and waning potential observed by Goto in auricular muscle."

In rabbit there is a left superior vena cava, while in man this vessel becomes rudimentary. In human embryo, there is a right and left duct of Cuvier and corresponding cardinal veins. In the later stage of the development the left duct of Cuvier becomes the oblique vein and proximal part of

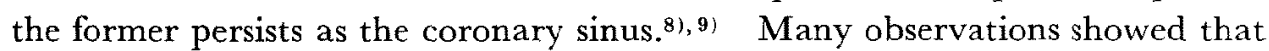
the region of the coronary sinus became often ectopic pacemaker. Zahn ${ }^{10}$ ) showed that in dogs a tachycardia was produced by the warming of the coronary sinus and was stopped by the cooling of this area. Eyster and Meek, ${ }^{111}$ after complete or nearly complete excision of the sinus node, produced the final permanent rhythm arisen from the coronary sinus. The area of the impulse 
formation was substantiated by the direct leads. Scherf ${ }^{12}$ ) showed that a coronary sinus rhythm was obtained by cooling or by clamping the sinus node. In man coronary sinus rhythm was produced experimentally, utilizing the bipolar pacing catheter in the ostium of the coronary sinus. ${ }^{13}$ ) In rabbit the automatic activities in the isolated strip of the left superior vena cava were at times observed without the action of aconitine when the strip of the vena cava contained the part of the coronary sinus. ${ }^{14)}$

In man the left superior vena cava may occasionally persist as in the case in rabbit. Recently, Hancock ${ }^{11)}$ reported that in the patient with persistent left superior vena cava coronary sinus rhythm was easily observed when the sinus activity was suppressed. He discussed that when the left superior vena cava persisted the coronary sinus portions of the A-V node also was more greatly developed and might have a pacemaker function which competed with and often dominated the sinus node. While, in the present experiment, it was shown that in rabbit the region of the left superior vena cava proximal to the heart as well as that of the coronary sinus became ectopic pacemaker. This may suggest that in such patient the muscle fibers in the left superior vena cava may have the capacity of ectopic pacemaker activity. These discussions are also consistent with the fact that embryologically the coronary sinus and the proximal part of the left superior vena cava are developed from the sinus venosus. ${ }^{9)}$

In the patient with persistent left superior vena cava the ectopic pacemaker formation in the left superior vena cava proximal to the heart may have the significance for the genesis of cardiac arrhythmias, especially supraventricular extrasystoles. ${ }^{16)}$

\section{SUMMARY}

The ectopic pacemaker formation by aconitine in the left superior vena cava proximal to the heart was studied in rabbit.

(1) Aconitine produced the automatic activities in the isolated strip of vena caval muscle.

(2) During the recovery from aconitine poisoning the action potentials with diastolic slow depolarization were recorded from the region of the left superior vena cava near the coronary sinus.

(3) In the in situ experiment, tachycardia was produced by the local application to the left superior vena cava near the coronary sinus.

(4) From the above-mentioned facts, it was suggested that aconitine induced the ectopic pacemaker in the left superior vena cava proximal to the heart and that the impulse from the ectopic focus spread not only to the peri- 
phery but also to the atrium.

\section{REFERENCES}

1. Mashiba, H., Yanaga, T., and Ito, M.: Kyushu J. Med. Sci. 14: 121, 1963.

2. Ito, M., Yanaga, T., and Mashiba, H. : Kyushu J. Med. Sci. 14 : 393, 1963.

3. Ito, M., Yanaga, T., Saeki, K., Arita, M., Ishihara, M., and Mashiba, H. : Jap. J. Physiol. $14: 439,1964$.

4. Saeki, K., et al. : unpublished observation.

5. Arita, M., et al.: in press.

6. Matsuda, K., Hoshi, T., and Kameyama, S. : Jap. J. Physiol. 9: 419, 1959.

7. Goto, M., Tamai, T., and Yanaga, T.: Jap. J. Physiol. 13 : 196, 1963.

8. Pattern, B. M. : Human embryology. The Blackiston Company, New York, 1953.

9. Keith, S. A.: Human embryology and morphology. Edward Arnold \& Co., London, 1933.

10. Zahn, A.: Arch. f. d. ges. Physiol. $151: 247,1913$.

11. Eyster, J. A. and Meek, W. J.: Am. J. Physiol. 61 : 117, 1922.

12. Scherf, D., Blumenfeld, S., and Yildiz, M.: Am. J. Cardiol. 10: 234, 1962.

13. Lancaster, J. F., Leonard, J. J., Leon, D. F., Kroetz, F. W., and Shaver, J. A. : Am. Heart J. $70: 89,1965$.

14. Ito, M., et al.: unpublished observation.

15. Hancock, E. W.: Am. J. Cardiol. 14: 608, 1964.

16. Mashiba, H. : Igaku no Ayumi : 56: 575, 1966. 